\title{
Perspectives on Insulin Stimulation of Glucose Transport
}

\author{
Richard D Sauerheber* \\ Palomar Community College, USA \\ *Corresponding author: Richard D Sauerheber, Chemistry, STAR Center, Palomar Community College, 1140 W. Mission Rd, San Marcos,
} CA 92069, USA.

To Cite This Article: Richard D Sauerheber. Perspectives on Insulin Stimulation of Glucose Transport. Am J Biomed Sci \& Res. 2019 - 4(5). AJBSR. MS.ID.000834. DOI: 10.34297/AJBSR.2019.04.000834

Received: 眥 August 06, 2019; Published: 眥August 21, 2019

\begin{abstract}
Although the mechanism by which insulin stimulates glucose transport proteins in the plasma membranes of insulin-sensitive cells has long been intensively studied, many details still remain controversial. The simplest, most energy-efficient mechanism that is consistent with most available data is described. The mechanism is counter to much published literature and is thus important to discuss.
\end{abstract}

\section{Introduction}

After the successful quantitative isolation of sufficiently purified insulin to inject into type I diabetic children by Banting, Best, Collip, and McCloud (1921) at the University of Toronto, insulin has been the most intensively studied of all hormones in endocrinology. During meal eating, insulin release from the pancreatic Islets of Langerhans lowers blood glucose by stimulating glucose uptake into tissues. The functional purpose of insulin, alone in causing glucose lowering that is opposed by 14 other hormones, is to store food breakdown products for energy to be supplied between meals when insulin levels return to basal. Insulin stimulates the formation of glycogen to store glucose for maintenance of blood glucose during times of fasting. Insulin stimulates lipid and protein synthesis while blocking glycogenolysis, proteolysis, and lipolysis.

It is well established that the glucose transporter is an integral protein that spans the cell surface membrane to provide a path for hydrophilic glucose molecules to pass through the hydrophobic bilayer without requiring expenditure of energy. Glucose concentrations outside the living cell are always higher than inside the cell, where glucose is quickly converted to glucose 6-phosphate by hexokinase, so that diffusion drives the entry process. The transporter has a recognition site that specifically only allows glucose to enter, and thus the uptake is referred to as facilitated diffusion. The number of glucose transporters in a cell vary with cell type, where erythrocytes which are not sensitive to insulin have the highest count at 200,000 transporters per cell [1]. The energy needs for cells to survive are met with the intrinsic complement of transporters for each cell type, as long as glucose is present in the extracellular fluid.

Insulin sensitive tissues such as liver, fat, and muscle, also contain insulin receptor proteins on the outer surface of the membrane with structures that specifically bind insulin when present in the fed state. In a cell with 15-30,000 receptors [2] spread around the cell surface, glucose transport rates increase within seconds after insulin binding occurs. Transport rates of non-metabolizable analogs of glucose are increased by insulin over the same dose response as for natural glucose so phosphorylation of transported glucose is not required. And there is no proof that ATP hydrolysis is required for the insulin stimulation of glucose transport through the bilayer where glucose transport itself is considered to be a facilitated transport process rather than an active transport process requiring energy.

Careful in vivo studies with labeled glucose indicate that insulin administration in mammals, where the hormone dilutes into the extracellular fluid to the concentration consistent with this fluid volume, stimulates glucose uptake quickly without requiring any uptake of insulin into the cell for full function. Every time a meal is consumed, insulin is secreted from pancreatic beta cells into the bloodstream that is quickly followed by increased glucose uptake into sensitive tissues. The uptake is extremely fast, as demonstrated by Wick and colleagues who detected the increased respiration of radiolabeled carbon dioxide from the incorporated glucose within minutes after insulin administration in intact animals [3]. 
It must be emphasized that the chief purpose for insulin to increase glucose uptake rates is not to provide additional energy for cellular function. Cells in the fasting state in the complete absence of insulin do not require additional glucose uptake simply because a meal is consumed. However, patients lacking insulin develop type I diabetes mellitus with blood glucose levels when prolonged leads to lethal diabetic coma due to lowered blood $\mathrm{pH}$ due to excessive formation of acid metabolites from excessive lipolysis. Prior to the use of insulin, diabetes was treated with prolonged fasting to extend patients' lives as long as possible.

Described here is the most logical attempt to delineate the mechanism by which insulin stimulates glucose transport. The vast literature examining the insulin stimulation of glucose transport in various insulin sensitive cells has produced much corroborating evidence. Differing published views developed from these data were analyzed and weighted for consistency with available facts and by considering the known physiologic purposes for the hormone.

\section{Energetics of Insulin Action}

It is generally held that insulin stimulates D-glucose uptake rates in insulin sensitive cells by approximately 2 -3-fold. This stimulation level at maximal insulin concentrations is obtained whether glucose concentrations are measured directly in extracellular fluid, or rather glucose derivatives such as 2-deoxy D-glucose are used to measure initial transport rates in intact cell suspensions. Glucose transport thus does not require phosphorylation of glucose from ATP hydrolysis. The stimulation rate achieves maximal levels within several seconds after addition into a cell suspension of insulin at maximally stimulating concentrations that are present in the bloodstream of humans and other mammals in the fed state. Non-metabolized glucose analogues are transported at the stimulated rate for the period of time when the transported molecules remain at a low concentration inside the cell. The initial stimulated rate is 2-3 times faster in the presence of insulin even when the transported molecules are not converted into a different product by intracellular phosphorylation and metabolism. Some studies however suggest that ATP phosphorylation of protein may be involved in the stimulation process.

\section{Intrinsic or Recruited Transport Proteins}

Two possibilities have been described by which insulin stimulates glucose uptake into cells, namely the activation of nascent transporters present in the membrane as a result of insulin binding, or that additional entire transport proteins become inserted into the membrane following insulin binding [4]. A large number of published articles promote the notion that insulin binding to its receptor stimulates glucose transport by causing the insertion or recruitment of whole transport proteins from inside the cell into the bilayer. On the other hand, additional studies confirm that insulin stimulation of transport shares features in common with the behavior of allosteric enzymes where activity is regulated by bound substances that simply induce a change in conformation of the protein.

One piece of evidence used to support the recruitment notion is that binding of cytochalasin B to glucose transporters is elevated after insulin stimulation. This however requires the assumption that the same number of molecules bind to transporters whether in the stimulated or basal state conformations and does not consider the possibility that transporters were already in the bilayer that could change conformation to become functional and then also bind cytochalasin B. Not only does cytochalasin B bind to multiple sites on the glucose transporter, the earliest use of the agent was to bind to cytoskeletal protein. Recent studies indicate a close link of cholesterol-rich membrane regions with the cytoskeleton and that cytochalasin B disrupts this arrangement [5]. Membrane conformational changes induced by insulin might expose such binding sites to cytochalasin B. Histologic microscopic evidence to find possible assembled transporters inside the cell that incorporate into the membrane after insulin binding occurs are hampered by the fact that the stimulation occurs so rapidly that it cannot be visually observed directly.

A protein insertion mechanism is far more complex and thus a less likely explanation for the action of insulin from both energy and time-dependence considerations. [1] Insulin activation of transport at the membrane level is extremely fast [6]. The incorporation of integral proteins into the hydrophobic plasma membrane bilayer however, such as occurs during chronic membrane turnover, requires a large expenditure of energy and substantial time. [7] The overall function of insulin during meal eating is to store energy, not to expend it. Typically eating three meals daily requires a simple mechanism for insulin to stimulate glucose uptake that does not deplete substantial amounts of energy. Protein incorporation into the membrane occurs during normal membrane turnover which is itself increased by the insulin stimulation of protein synthesis, but the process is highly energy dependent and slow compared to the glucose transport stimulation by insulin that occurs in mere seconds. The return to basal transport rates is also a rapid process. The quick fully reversible stimulation is inconsistent with insertion into the bilayer followed by the removal again of assembled transporters from the bilayer back into intracellular regions. Recycling of inserted transporters to again return to a basal state would also require hydrolysis of ATP for energy to remove the integral proteins back into a vesicular arrangement inside the cell.

Expenditure of energy for merely the process of allowing glucose to enter the cell from concentrated extracellular fluid is counter to the physiologic purpose of insulin, which is to conserve energy. Facilitated diffusion by the glucose transporter is understood to be distinct from other active transport processes that require ATP consumption. Facilitated diffusion by name relies on the concentration gradient for transport to simply chemically occur, rather than requiring expenditure of energy. Also, synthesizing new 
membrane, as in membrane turnover, is a process that requires vast amounts of energy, to insert structures into the hydrophobic bilayer that are soluble in intracellular fluid. There is no evidence that insulin somehow turns glucose transport, a facilitated diffusion non-energy-requiring process, into a system that would be characterized as an active transport process that requires ATP.

Insertion or translocation of assembled proteins to become integral membrane proteins, from the aqueous intracellular environment into the bilayer, is highly energy-dependent and is not a process that would likely occur within seconds. Although lateral migration of integral proteins within the fluid lipid bilayer was initially characterized as rapid, on the order of seconds, incorporation of such integral membrane protein molecules as in membrane assembly is not rapid. Studies attempting to determine if insulin stimulation of glucose transport actually involves insertion of proteins into the membrane every time a meal is consumed are hampered by the fact that incubation with insulin is not easily limited to seconds duration while cell-free preparations are made or while tissue fixation is undertaken for microscopic study. A key study for example incubated cells for 30 minutes to reveal what were believed to be apparent shifts in staining of transport proteins visible microscopically between intracellular and surface membrane locations. However, histologic studies have not shown that any intracellular transporters to be inserted are distributed around the cell surface in any particular relationship with the cellular distribution of insulin receptors.

The anatomy of mammalian fat cells examined microscopically also support the intrinsic transporter stimulation mechanism The fat cell nucleus, mitochondria, and ribosomes responsible for protein synthesis are sequestered in a small pocket of cytoplasm. The entire cell surface membrane surrounds the bulk internal mass of triglyceride is barely separated from the bilayer by only a very thin ring of cytoplasm without significant subcellular organelles. Further, finding that homogenized cell preparations have a higher intracellular protein content in the plasma membrane fraction after insulin treatment does not prove that glucose transporters were inserted into the surface membrane. It is extremely difficult to remove all intracellular membrane fragments from plasma membrane fractions in homogenized preparations. And the fact that insulin affects the structure of the membrane surface, alters ion and amino acid transport, and activates phosphorylation and stimulation of lipid, protein, and glycogen synthesis while separately inhibiting lipolysis, proteolysis, and glycogenolysis inside cells, it is not surprising that insulin-treated cells might exhibit a differing content of intracellular membrane fragments in a plasma membrane fraction compared to the basal state.

Although vesicle insertion into the plasma membrane from intracellular locations is known, as in nerve conduction across a synapse, it is difficult to justify entire vesicle fusion with incorporation of integral protein transporters into the plasma membrane merely to enhance uptake rates into cells that already contain functioning intrinsic transporters. Synapses slow membrane conduction and exist in part to ensure one-way direction of travel of an electrical impulse. There is no requirement to recycle the contents of these vesicles because the contents are extruded from the cell. This is very much different than simply increasing and then decreasing again the rates of glucose uptake between fed and then postprandial states. And the purpose of vesicle uptake into membranes is to release the soluble vesicle contents. Inserting a hydrophobic integral protein transporter of complex structure to span the entire bilayer is a much different process.

The argument that additional transporters might be necessary for increased ATP production to be available for the metabolic steps in the synthesis of lipid, protein, and glycogen is not necessary when glucose uptake rates are already elevated by the stimulated intrinsic membrane transporters within seconds. Insertion of transporters as a chronic process to support long-term metabolic and membrane structural needs in membrane turnover is of course physiologic. Insulin stimulation can be impaired by poisoning intact cells that blocks ATP synthesis, suggesting it may be an energy requiring process. But this may be due to the need for an intact functioning cell membrane with the high ATP demanding sodium/potassium exchange to maintain normal membrane protein conformation, rather than any specific need for ATP hydrolysis in the stimulation process.

A few key experiments have been conducted to distinguish whether insulin causes the insertion of new additional transporters into the bilayer, or rather increases the activity of transporters already in the membrane prior to insulin addition. Adipocyte glucose transporters on cell surfaces were selectively inhibited in activity by treatment with fluorescein isothiocyanate that does not penetrate cells. It was indeed found that the fold stimulation of transport under these conditions is identical to the normal stimulation effect. This is difficult to explain if insulin actually caused the insertion into the membrane of fully functional transporters that were not altered by the drug treatment [8]. Although this work was challenged in experiments incubating cells for an extensive time (45 minutes) [9] the data under our careful conditions are clear.

Additional tests of the hypothesis, that the mechanism involves a relatively direct stimulation of transporters subsequent to insulin binding, used membrane structural perturbants to find how sensitive the stimulation process is to such changes. Agents that broadly increase the fluidity of the lipid bilayer in adipocytes indicate that large perturbations in the lipid bilayer that inhibit glucose uptake rates do notaffect the insulin stimulation mechanism. Ethanol concentrations from 0.3 to $1.5 \mathrm{M}$ that drastically increase the fluidity of the fat cell plasma membrane and decrease basal glucose transport rates by up to $60 \%$ do not impair insulin function. 
Insulin stimulates glucose uptake 2-3-fold over this entire ethanol concentration range. This indicates a close relationship between the insulin receptor and insulin-sensitive glucose transporters, where gross alterations in the lipid structure surrounding these proteins do not impair the stimulation mechanism. Similar findings were reported for the actions of benzyl alcohol up to $40 \mathrm{mM}$ where the lipid bilayer was substantially perturbed but without effect on the insulin inhibition of glycerol release in fat cells. Further, benzyl alcohol at $25 \mathrm{mM}$ that inhibits glucose transport rates by $50 \%$ leaves the insulin stimulation of hexose transport intact at any insulin concentration added and for any time point during the stimulation process. Neither the maximal stimulation nor the time-dependence of the stimulation of transport caused by insulin are affected, while basal transport rates are substantially inhibited by the increased bilayer fluidity. This means that the conformation change on the receptor that occurs upon insulin binding is sensed by transporters in a quickly and regionally highly connected manner.

The temperature dependence of the insulin stimulation of transport has been thoroughly investigated $[10,11]$. The optimum temperature for maximal stimulation is as expected at physiologic temperature, $37 \mathrm{oC}$. But even at a low temperature of $25 \mathrm{oC}$ insulin stimulates glucose transport significantly, by $44 \%$. A complex mechanism involving insertion of proteins into the bilayer matrix would be expected to be drastically inhibited at this temperature. Basal transport rates are remarkably steady over the broad range from 25 to $430 \mathrm{c}$, because the transport protein does not catalyze a chemical reaction but merely holds a pore in place through which glucose can diffuse.

It must be pointed out that the phenomenon of conformation change causing change in function of proteins is well known, as with allosteric enzymes that change rates after regulators bind to sites distant from the active site, or oxygen-carrying hemoglobin that changes conformation as a function of the amount of oxygen bound to the heme prosthetic group. The allosteric phenomenon is also known where one protein interacts with another protein to change its conformation, such as the stimulation of lipase by protein kinase after being activated by the binding of cyclic AMP. It is thus not surprising to consider that the glucose transporters in a large molecular complex in the membrane can sense the altered conformation of the insulin receptor after insulin binds the receptor on the cell surface. Consistent with changes in protein conformation being responsible for the stimulation mechanism, it must be noted that specifically hydrolyzing sialic acid residues from the membrane surface (personal communication, T. Ciaraldi), or merely lowering extracellular fluid $\mathrm{pH}$ from 7.4 to 7.2 obliterate the insulin stimulation of glucose transport. Cuatrecasas [12] reported that highly purified neuraminidase removes freely exposed sialic acid residues without affecting insulin binding to its receptor or the complete metabolism of radiolabeled glucose to carbon dioxide in fat cells. The removal somewhat alters cellular glucose uptake and 3-0-methyl glucose transport rates but completely obliterates the insulin stimulation of transport, in addition to obliterating the insulin inhibition of lipolysis.

\section{Proposed Model}

The best concept that is consistent with these data is that insulin receptors and multiple glucose transporter proteins exist in a complex organized structure in the bilayer first proposed by Cuatrecasas in 1974 [13]. This protein complex could be protected within a membrane lipid arrangement maintaining its stability within an otherwise more fluid matrix. Cholesterol known bilayer ordering would be a possible component in such a structure. The substantial conformational change in the receptor after insulin binding would compress or rearrange the ordered unit to cause transporters to change shape. This is consistent with the principle of Occam's razor, being a direct and simple allosteric mechanism, consistent with X-ray crystallographic studies of allosteric proteins where 3-dimensional allosteric changes exhibit time-dependence comparable to that for insulin stimulation of transport [14,15].

\section{plasma membrane}

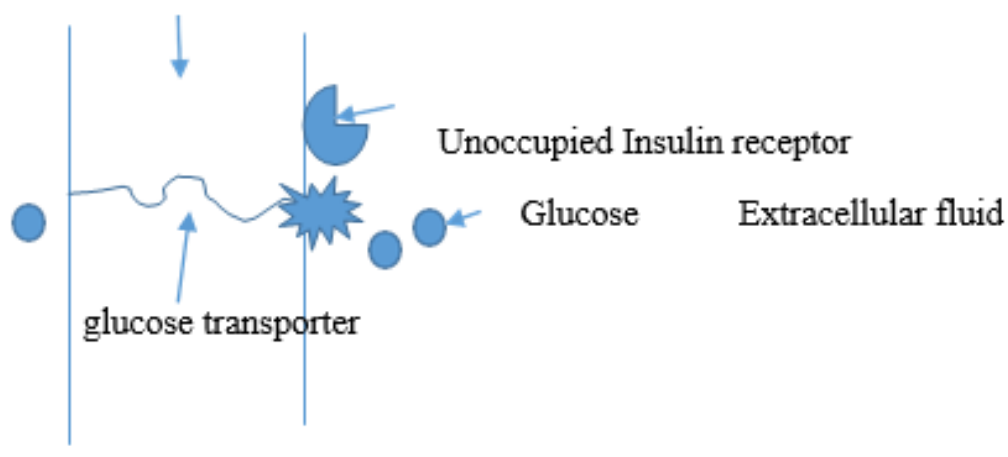

Figure 1A: Representation of an insulin-sensitive glucose transport integral membrane protein in the basal state conformation. Here insulin is not bound to the receptor which is an extrinsic membrane protein that specifically only binds insulin. The path length glucose molecules are required to travel through the membrane into the cell is longer than for the insulin stimulated conformation in $1 \mathrm{~B}$. The transporter structure at the surface of the membrane only allows glucose specifically to enter the interior region of the transporter. 


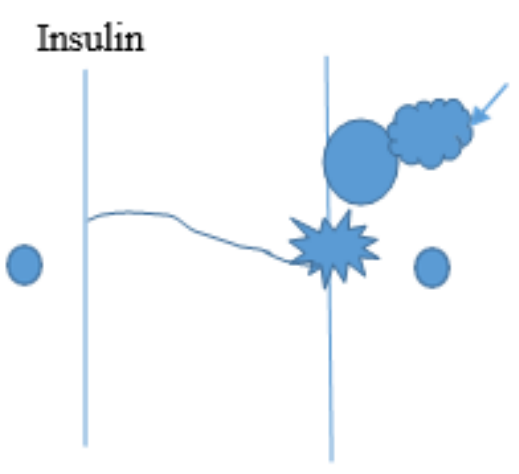

Figure 1B: Representation of the glucose transporter after undergoing a conformational change caused by insulin binding to its nearby receptor, in a manner analogous to an allosteric enzyme in response to an agonist. The path length required to travel through the membrane for glucose molecules to enter the cell is less than for the basal state protein conformation. Approximately twice as many glucose molecules enter the cell per unit time than for the basal state.

Figure $1 \mathrm{~A} \& 1 \mathrm{~B}$ indicates a crude representation of such an allosteric regulation of glucose transport proteins upon binding of insulin to the cell surface receptor. The diagram is purposely simplistic to emphasize that no accurate depiction of the complex 3-dimensional integral protein in its native configurational arrangement in the bilayer is known. Since glucose is polar and fully water soluble it must be that the internal hydrophilic regions of the glucose transporter are sequestered from the hydrophobic surroundings to allow a passageway for glucose to travel through the membrane. It is not difficult to consider that a conformational change in the transport protein could alter the path length of hydrophilic domains in the bilayer so that glucose transport rates are increased. The transport rate present in the basal state would reflect the glucose travel path length being longer than the insulinstimulated conformation with a shortened path length and hence an increased rate at which glucose molecules gain entry into the cell.

Some have suggested that insulin acutely stimulates both the intrinsic activity of transporters in the membrane and also the incorporation of additional transporters into the membrane for its immediate effects on glucose uptake. Although increased protein synthesis is a more chronic effect of insulin stimulation, it is inappropriate to completely eliminate the more simple mechanism for the acute effects of the hormone.

An alternative explanation might be that additional transport proteins are already present in the membrane that only activate when insulin binds the receptor. In this way, the conformation change would involve simply opening the entrance to the channel, allowing glucose to enter at twice the rate. If the entrance conformation were the rate-limiting step, then two entrance channels would double the uptake rate for a single transporter path in the bilayer. If half the transporters were associated with the insulin receptor and sensitive to insulin in this way, or if the original number of transporters had two entrance sites open in the presence of insulin, this would double the uptake rate, again without requiring energy to be expended every time a meal is consumed.

\section{Significance}

Much evidence indicates that the most common form of diabetes, type II formerly known as 'adult onset' or obesity associated, is typically caused by over-nutrition, where no new cases were documented during wartime rationing [16]. However, the insulin stimulation mechanism could be directly inhibited in some cases in man, including the rare genetic disease sialidosis [17], or during severe acidosis. These conditions can cause hyperglycemia not correctable with fasting, being a true membrane insulin resistance due to an altered structure of the insulin stimulating apparatus.

\section{Acknowledgment}

This analysis stems largely from much research previously conducted under the financial support of the National Institutes of Health (1981-1984) and the California Metabolic Research Foundation (1973-1990). Thanks go to Palomar students for their interest and Kalvin McCallum for examining the manuscript.

\section{References}

1. Amelie Montel-Hagen, Lionel Blanc, Myriam Boyer Clavel, Chantal Jacquet, Vidal M et al. (2008) The Glut1 and Glut4 glucose transporters are differentially expressed during perinatal and postnatal erythropoiesis. Blood 112(12): 4279-4738.

2. Blackard WG (1977) Insulin Receptors. Medical College of Virginia Quarterly 13(1): 12-16.

3. Wick AN, Drury DB, Bancroft RW, MacKay EM (1951) Action of insulin on the extrahepatic tissues. JBC 188: 241-249.

4. Czech MP (1981) Insulin action and the regulation of hexose transport. Diabetes 29(5): 399-409.

5. Romanenko VG, Roser KS, Melvin JE, Begenisich T (2009) The role of cell cholesterol and the cytoskeleton in the interaction between IK1 and maxi-K channels, Am J Physiol Cell Physiol 296(4): 878-888.

6. Ciaraldi T, Olefsky J (1982) Kinetic relationships between insulin receptor binding and effects on glucose transport in isolated rat adipocytes. Biochemistry 21(14): 3475-3480.

7. Mueckler M, Thorens B (2013) The SLC2 GLUT family of membrane transporters Molecular Aspects of Medicine. Mol Aspects Med 34(2-3): 121-138.

8. Hyslop P, Kuhn C, Sauerheber R (1985) Insulin stimulation of glucose transport in isolated rat adipocytes: functional evidence for insulin ac- 
tivation of intrinsic transporter activity within the plasma membrane. Biochem J 232(1): 245-254.

9. Goto Y, Suimda Y, Flanagan J, Robinson F, Simpson I, et al. (1992) Effects of Fluorescein Isothiocyanate on Insulin Actions in Rat Adipocytes. Archives of Biochemistry and Biophysics 293(2): 224-230.

10. Sauerheber R, Kuhn C, Hyslop P (1984) Membrane Structural/Functional Properties of Adipocytes from Normal and Steptozotocin Diabetic Rats. Diabetes 33(3): 258-265.

11. Hyslop P, Kuhn C, Sauerheber R (1984) Temperature optimum of insulin-stimulated 2-deoxy-D-glucose uptake in rat adipocytes. Correlation of cellular transport with membrane spin and fluorescence label data. Biochem J 218(1): 29-36.

12. Cuatrecasas P, Illiano G (1971) Membrane Sialic Acid and the Mechanism of Insulin Action in Adipose Tissue Cells. Journal of Biological Chemistry 246(16): 4938-4946.
13. Cuatrecasas P (1974) Membrane Receptors. Annual Review of Biochemistry 43: 169:214.

14. Martin DW, Mayes PA, Rodwell VW (1982) Harper's Review of Biochemistry. (18 $8^{\text {th }}$ Edn.) Lange Medical Publications Los Altos CA.

15. Hyslop PA, Kuhn C, Sauerheber R (1987) Insulin Stimulation of Adipocyte Membrane Glucose Transport: a graded biologic response insensitive to bilayer lipid disordering. Biochemical Pharmacology 36(14): 2305-2310.

16. Sauerheber R (2018) Analysis of Type II Diabetes Mellitus; the Metabolic Condition and its Proper Correction. J Curr Chem Pharm Sc 8(2): 1-15.

17. Dridi L, Seyrantepe V, Fougerat A, Pan X, Bonneil E, et al. (2013) Positive regulation of insulin signaling by neuraminidase 1. (Open Access) Diabetes 62(7): 2338-2346. 